\title{
EDUCACIÓN POPULAR AMBIENTAL Y RESISTENCIA CAMPESINA AL EXTRACTIVISMO EN EL MUNICIPIO DE SAMACÁ-BOYACÁ
}

\section{POPULAR ENVIRONMENTAL EDUCATION AND CAMPESINE RESISTANCE TO EXTRACTIVISM IN THE MUNICIPALITY OF SAMACÁ-BOYACÁ}

\section{Natalia Elisa Ramírez Hernández ${ }^{1}$ \\ Laura Viviana Hernández Rojas ${ }^{2}$ \\ Wilmer Yesid Leguizamón Arias ${ }^{3}$}

Universidad Pedagógica y Tecnológica de Colombia UPTC

$1 \quad$ Abogada Universidad Santo Tomas, especialista en Derecho Penal y Ciencias Forenses Universidad Católica de Colombia, Magister en Derechos Humanos Universidad Pedagógica y Tecnológica de Colombia UPTC, estudiante de Maestría en Filosofía Universidad Nacional de Quilmes- Argentina, Investigador Junior (Colciencias) adscrito al grupo de investigación Justicia Social Primo Levi. Correo electrónico: nataliaramirezabogada@gmail.com, natalia.ramirez01@uptc.edu.co,

Natalia Elisa Ramirez Hernandez código orcid https://orcid.org/0000-0001-6093-025

$2 \quad$ Licenciada en Educación Preescolar, especialista en Pedagogía de los Derechos Humanos y estudiante de maestría en Derechos Humanos de la Universidad Pedagogia y Tecnológica de Colombia, investigadora adscrita al grupo de investigación justicia social Primo Levi de la Universidad Pedagógica y Tecnológica de Colombia, correo electrónico lauravivianaz.321@gmail. $\underline{\mathrm{com}}$

Wilmer Yesid Leguizamon Arias código orcid https://orcid.org/0000-0003-1596-0277

3 Abogado Universidad Pedagógica y Tecnológica de Colombia, Magister en Derecho Universidad Nacional de Colombia, estudiante de doctorado en Derecho Público- Universidad Santo Tomas, docente de las universidades Juan de Castellanos y Universidad Pedagógica y tecnológica de Colombia UPTC, Investigador adscrito a los grupos de investigación Hugo Grocio y Primo Levi. Correo electrónico: wyleguizamon@jdc.edu.co, wilmesid@gmail.com

Laura Viviana Hernandez Rojas código orcid https://orcid.org/0000-0002-4198-0573 


\section{RESUMEN}

Expone una experiencia de educación popular en el contexto de las conflictividades socio ambientales derivadas de la extracción de carbón en el municipio de Samacá Boyacá. Para ello en principio se analizan los planteamientos básicos de la educación popular, la lucha frente al cambio climático y su aplicación en la educación ambiental. Posteriormente, en un segundo apartado se expone brevemente el proyecto de investigación titulado "Gobernanza ambiental y movimientos sociales casos de defensa del bosque alto andino en Boyacá" y se reflexiona en relación al municipio de Samacá Boyacá, finalmente, se presentan los resultados de investigación obtenidos donde la educación popular se vislumbra como una oportunidad para la construcción colectiva de soluciones concertadas entre el movimiento campesino emergente y las empresas que se dedican a la extracción de carbón y coquización de carbón que interactúan en un mismo ámbito territorial.

\section{PALABRAS CLAVE}

Educación Popular, Educación Ambiental, Gobernanza Ambiental, Movimientos Campesinos, Extractivismo

\section{ABSTRACT}

This article presents a popular education experience in the context of socio-environmental conflicts arising from the extraction of coal in the municipality of Samacá Boyacá. To do this, the basic approaches of popular education, the fight against climate change and its application in environmental education are analyzed in principle, then in a second section the research project entitled "Environmental governance and social movements defense cases is briefly presented. of the high Andean forest in Boyacá "and is reflected in relation to the municipality of Samacá Boyacá, finally, the results of research obtained where popular education is seen as an opportunity for the collective construction of concerted solutions between the emerging peasant movement and the companies dedicated to the extraction of coal and coal coking that interact in the same territorial area..

\section{KEYWORDS}

Popular Education, Environmental Education, Environmental Governance, Peasant Movements, Extractivism.

\section{SUMÁRIO}

Este artigo apresenta uma experiência de educação popular no contexto de conflitos socio ambientais decorrentes da extração de carvão no município de Samacá Boyacá. Para isso, as abordagens básicas da educação popular e sua aplicação na educação ambiental são analisadas em princípio; em seguida, uma segunda seção expõe brevemente $\mathrm{o}$ projeto de pesquisa intitulado "Governança ambiental e casos de movimentos sociais de defesa da floresta de alta endino em Boyacá" e se reflete em relação ao município de Samacá Boyacá, finalmente, são apresentados os resultados de pesquisas obtidas onde a educação popular é vista como uma oportunidade para a construção coletiva de soluções concertadas entre o movimento camponês emergente e as empresas envolvidas na extração carvão e coque de carvão que interagem na mesma área territorial.

\section{PALAVRAS CHAVE}

Educação Popular, Educação Ambiental, Governança Ambiental, Movimentos Camponeses, Extrativismo

\section{INTRODUCCIÓN:}

La definición de Educación Popular surge con fuerza en américa latina a partir de los años 60 en un contexto de agitación social en el cual se escucharon demandas de diferentes movimientos sociales que reclamaban un orden social más justo. (Calixto, 2010, pág. 31) Sus postulados se encuentran contenidos en su mayoría en la obra de Paulo Freire, que 
de forma precisa y aún vigente estableció que los oprimidos debían descubrir su realidad y liberarse atreves de la trasformación de sus entornos en una doble vía donde educador y educado aprenden de forma recíproca y a partir del dialogo de saberes trasforman la realidad. (Freire, 1988)

La educación popular excluye dentro de sus fronteras las tradiciones pedagógicas preexistentes, mueve la educación a las calles y concibe al educando como portador de conocimientos validos que aporta al educador, pues separa y recoge un conjunto de vivencias que le permiten orientar sus propias necesidades hacia una pedagogía más integral, de esta forma se excluyen modelos pedagógicos que promueven o reproducen una organización de poder concentrado y organicista.

Esta distinción permite a la educación popular ser parte activa de transformaciones sociales pues su principal función es emancipar, liberar, transformar, lo que va de la mano con los fines perseguidos por los movimientos sociales en sus luchas, tal como sucede con el movimiento ambientalista.

Un fenómeno creciente a nivel regional, nacional y local es la conciencia ambiental que se acentúa como respuesta a las prácticas extractivas de las últimas décadas. En este contexto, las comunidades han experimentado diferentes mecanismos institucionales y no institucionales de gobernanza ambiental, que van desde las consultas populares y referéndum hasta movilizaciones sociales no violentas, pasando por el uso de acciones judiciales y administrativas.

Bajo este contexto, el proyecto de investigación "Gobernanza ambiental y movimientos sociales: casos de defensa del bosque altoandino en Boyacá" creado con código SGI 2387, desarrollado por los Grupos de Investigación Hugo Grocio de la Facultad de Ciencias Jurídicas y Políticas Internacionales de la Fundación
Universitaria Juan de Castellanos y Primo Levi de la Facultad de derecho y ciencias sociales de la Universidad Pedagógica y Tecnológica de Colombia UPTC, tomo como parte del objeto de estudio al municipio de Samacá Boyacá, teniendo en cuenta que en su jurisdicción se encuentra ubicada en el distrito de páramos Rabanal de Cundinamarca y Boyacá; considerado un macizo aislado por estar distanciado a más de $10 \mathrm{~km}$ de sus complejos vecinos (nacimiento del Río Bogotá, SFF Iguaque y Páramo de Guerrero), lo que le da una gran importancia ecosistémica, (IAvH; CAR; Corpochivor; Corpoboyacá; Gobernación de Boyacá, 2016) adicionalmente alrededor del complejo se encuentra un conjunto de ecosistemas de alta montaña, que van desde bosque andino, en las alturas inferiores, hasta páramo, en las mayores alturas. (Cárdenas, 2018)

Teniendo en cuenta lo anterior se analiza la evolución en los procesos de gobernanza del agua dentro del distrito de riego asociación de usuarios del distrito de adecuación de tierras de Samacá - ASUSA, haciendo especial hincapié en la transformación sufrida a partir de las afectaciones ambientales causadas por la actividad extractiva de carbón y el servicio de hornos de coquización sobre los ecosistemas de paramo y bosque altoandino que surten al distrito de riego y otros acueductos rurales. El conflicto causado a partir de la afectación al recurso hídrico interpeló a la asociación de usuarios respecto a la protección del medio ambiente y el camino que deberían seguir de cara a la conservación y cuidado de las fuentes hídricas en su territorio.

En efecto, por muchos años los habitantes de Samacá han visto la actividad minera como su principal fuente de ingreso y han convivido con los impactos ambientales producto de esta actividad; pero el escalamiento de tales daños al punto de poner en riesgo la sustentabilidad del recurso hídrico ha cuestionado la posición 
que se venía asumiendo respecto a la actividad minera, este fenómeno se puede ver condensado en el caso seleccionado del distrito de adecuación de tierras de Samacá - ASUSA y las trasformaciones de sus procesos de gobernanza del agua.

A partir de ello fue posible describir un panorama socio jurídico en relación a los mecanismos institucionales y no institucionales definidos para la defensa del medio ambiente por parte de la ciudadanía en general y las organizaciones socio ambiental en particular y formular propuestas en torno a los mecanismos de participación ciudadana en materia ambiental, ajustadas a la realidad socio ambiental que actualmente vive el Estado colombiano de manera general y el Departamento de Boyacá de manera particular.

En otros términos, el estudio permitió contrastar y analizar de forma critica el diseño institucional de los instrumentos de participación ciudadana así como los medios de control y auto control administrativo y judicial, usados por las organizaciones sociales para reclamar la protección de sus derechos frente a la industria minera y de hidrocarburos, cuando amenazan ecosistemas de bosque altoandino en el Departamento de Boyacá.

A partir de esta experiencia se desarrolló un proceso de educación popular con las personas del distrito de adecuación de tierras de Samacá - ASUSA, quienes nos compartieron sus mecanismos para ejercer la gobernanza del agua, su territorio tanto en la zona minera como en la zona de paramo y sus preocupaciones en relación al futuro del agua en su municipio, esto nos permitió compartir con ellos una cartilla didáctica que obedece a las necesidades expresadas por la comunidad, la cual comienza su fase de socialización.

El trabajo de investigación se desarrolló con el modelo de investigación acción participativa de Orlando Fals borda, con una metodología cualitativa que nos permitió una aproximación a la comunidad con el fin de describir y comprender las formas de gobernanza ambiental para lo cual se elaboró una fase inicial de diagnóstico, posteriormente se delimito la población y finalmente se abordó la problemática a partir de la educación popular.

\section{EDUCACIÓN POPULAR AMBIENTAL}

Los procesos de educación popular se encuentra en gran medida influenciado por las necesidades sociales y las demandas por alcanzar mejores condiciones de vida que acompañan a los movimientos sociales, al presente aparte desarrolla brevemente los postulados básicos de la educación popular y su aplicación en la lucha por la defensa del medio ambiente y el territorio.

\subsection{Educación popular}

La educación popular busca evidenciar las voces que han sido silenciadas a través de la historia, se concibe como esperanza para todos aquellos marginados de las decisiones colectivas. Para paulo Freire el punto de partida para formar en educación popular se cimienta en la pedagogía crítica ${ }^{4}$. Así las cosas la educación popular asume como propios algunos postulados de la pedagogía critica como la participación social, la comunicación horizontal, la significación de imaginarios simbólicos, la humanización de los procesos educativos, la contextualización de los procesos y la transformación de las realidades sociales. Con estos elementos la educación popular cuenta con el potencial necesario para transformar la vida de cualquier persona en virtud a sus consignas liberadoras y emancipadoras, pero en especial busca generar cambios significativos en la vida de las personas

$4 \quad$ Movimiento educativo que propone el análisis crítico y reflexivo dentro de la escuela, y pretende liberarse de la pedagogía bancaria o conductual que busca limitar la posibilidad de creación de los propios conocimientos fomentando la reproducción sin análisis ni comprensión de los temas que se enseñan con el fin de repetir lo que ya se ha dicho. 
marginadas, los oprimidos 5 .

Este tipo de educación no está condicionada a las aulas pues es precisamente dirigida a un conglomerado humano que quiera hacer parte de proceso de educación liberadora, separada de prejuicios conductuales y encargada de transformar la ideología de la alienación por un pensamiento emancipador de realidades sociales, invitado a crear nuevas formas de conocimiento crítico motivador, generando un cambio en el que se incluye a todas las personas pero en especial a las que no poseen recursos económicos, pues la tarea con el compromiso ético-político de educar en doble vía, desde la experiencia, desde la lucha constante por sobrevivir en un país limitado en oportunidades para las personas de bajos recursos económicos, es el fin último de la educación popular. (Freire, 1988)

La trascendencia de la pedagogía crítica en la educación popular radica pues en su afán por la transformación social a partir de la enseñanza. El significado de emancipación progresiva desde la mirada de construcción del otro sin perder de vista los espacios, los contextos, los imaginarios y los proyectos de vida de cada ser humano, las diferencias, las competencias de los individuos como complementos fundamentales en la sociedad.

A partir de lo anterior se introduce el énfasis en el diálogo para la educación informal, más que curricular y basada en contenidos culturales en donde lo más destacado es la praxis en el ejercicio educativo incorporando la pedagogía de la pregunta, para la comprensión critica de las realidades sociales; esto obedece a la consideración de Freire en la cual los opresores son solo educadores autoritarios que niegan la solidaridad entre el acto de educar y el acto de ser educados por los educandos. (Freire, 1988)

$5 \quad$ Freire lo explicaba en su libro la pedagogía del oprimido pues decía que la educación popular estaba pensada para las personas más pobres y de color porque siempre han sido las más marginadas por la sociedad,
Otro pilar de la pedagogía popular de Freire, radica en la necesidad de romper con el paradigma deshumanizante ${ }^{6}$, lo que le forjó el ambiente propicio para el constructo de nuevos movimientos sociales en América Latina, generándose todo un abanico de enfoques sobre tópicos como la educación, el trabajo comunitario, el género, el poder local y el medioambiente. Con estos elementos Freire inicia una lectura crítica de la realidad social, de las injusticias que observa a su alrededor ${ }^{7}$ y que a su juicio se generan por el sistema capitalista que a su vez reproduce la idea de orden social en la cual el sistema escolar juega un papel fundamental. (Muñoz, 2017)

De lo anterior se desprenden tres formas de entender la educación; la primera referida a la práctica social de la cultura, la segunda relacionada con los escenarios inducidos por el sistema educacional y la tercera en referencia a las acciones propias de cada sujeto que auto determinan su destino. De la suma de estos tres elementos nace el término popular, por tanto en el campo pedagógico se permite avanzar en el fortalecimiento de las luchas dinámicas de movimientos sociales y empoderamiento de personas vulnerables en el ejercicio de sus derechos fundando un impacto positivo y garantizando unas condiciones mínimas de dignidad humana. (Arciga, 2007)

La praxis y las reflexiones para transformar el mundo a través del diálogo de saberes, las dinámicas educativas y emancipadoras analizadas desde una perspectiva cultural es lo más significativo de los procesos de

$6 \quad$ La cultura y costumbres populares, el entorno en el cual se desarrolla el ser humano desde el inicio de su vida tales como la dependencia económica y las situaciones de vida en comunidad etc.

$7 \quad$ En la época en que se empieza a hacer notoria la alfabetización de Paulo Freire, pues era indispensable saber leer y escribir para votar en las elecciones de Brasil, en vista de ello Freire aprovecha la oportunidad para iniciar un proceso colectivo de cultura popular forjando una organización comunitaria para el trabajo formativo del campesinado, lo que permite dar un gran avance en la educación popular y liberadora. 
aprendizaje. Si bien es cierto Freire desarrolla la teoría de educación popular desde las clases populares de Latinoamérica y se centra en generar una transformación emancipadora del poder hegemónico precisamente partiendo de la práctica de experiencias de los oprimidos también es cierto que la educación esta provista de una función liberadora de lo contrario no se podría considerar educación en palabras de Freire:

"Enseñar no es transferir conocimiento, sino crear las posibilidades para su propia producción o construcción" (Freire, 2006, pág. 47)

Así mismo critico los métodos tradicionales de educación al considerar que no cumplían con la consigna de educar para empoderar en el contexto de la educación liberadora, el mismo Freire sostuvo:

"En el fondo, los métodos tradicionales de alfabetización son instrumentos "Domesticadores", casi siempre alienados, y además alienantes" (Freire, 2007, pág. 13)

Las lógicas de dominación propias de los métodos tradicionales de educación, se mantienen vigentes y son aplicadas en las aulas por los educadores de forma general, no por voluntad propia, sino por exigencia del estado convirtiendo así al educando en un objeto de manipulación moldeable que responde a una estructura de poder dominante dentro de la sociedad.

La educación popular se contrapone a los modelos de educación tradicional e invita a la integración del individuo con la realidad, insta a la pérdida del miedo a la libertad, la iniciación en el pensamiento político, la conciencia como sujeto de su propia historia, la capacidad de analizar críticamente las acciones propias y de los demás y la concienciación de un diálogo interpersonal. En este sentido Freire ubica este último punto como el despertar de la conciencia que se logra por medio de la comprensión de la constante lucha por parte de masas oprimidas, y la realización de una aceptación propia y de los demás dentro de la naturaleza. (Jiménez, 2014)

El valor de la palabra en Freire es un derecho intrínseco de todo ser humano, por ello, una verdadera educación parte del diálogo, un proceso de educación en doble vía donde la conversación estimula un proceso de reflexión, de esta forma se conduce a encontrar el conocimiento por sí mismo al generar el entorno para producir un conocimiento propio estimulando la crítica y la reflexión generando "sujetos" de su propio destino en palabras de Freire:

"El diálogo no impone, no manipula, no doméstica, no esloganiza" (Freire, 1988, pág. 205)

Desde ese postulado se desprende toda una crítica el sistema actual de escolarización que no ha logrado obedecer a las concepciones de la educación liberadora. Aplica verticalidad continua entre educador y educando, separa todo intento de diálogo que permita avanzar en la construcción de conocimiento, lo que se conoce como educación bancaria, en otras palabras, actualmente los modelos educativos aplicados obedecen al autoritarismo condicionado por calificaciones que miden al educando de acuerdo a las habilidades cognitivas adquiridas, impuestos por conductas en donde aún se cree que la "letra con sangre entra" o concepciones como que el educador es quien disciplina y el único poseedor del conocimiento, "quien tiene la verdad absoluta", desfigurando por completo la condición humana del educando.

En contraposición a la educación bancaria, la educación que Paulo Freire propone es: "Eminentemente problematizadora, fundamentalmente crítica y virtualmente liberadora." (Freire, 2007, pág. 18) Aplicada la educación popular se encuentra con que 
nadie educa a nadie, nadie se educa solo, los hombres se educan entre si y en esa medida el diálogo de saberes se vuelve fundamental para el aprendizaje comunitario que esta mediatizado por el mundo. (Freire, 2007, pág. 18)

En su obra política y educación Freire describe a través de la historicidad del ser humano su concepto de educación afirmando que los seres humanos por naturaleza están vinculados a alguna práctica educativa no necesariamente ligada a la escolaridad. En palabras de Freire:

"La educación como formación, como proceso de conocimiento, de enseñanza, de aprendizaje, ha llegado a ser a lo largo de la aventura de los seres humanos en el mundo una connotación de su naturaleza gestándose en la historia, como la vocación de humanización." (Freire, 1996, pág. 24)

En conclusión la educación popular es una praxis, reflexión y acción del ser humano sobre el mundo para transformarlo, teniendo como herramienta principal la construcción de su propia realidad a partir de la experiencia de vida, para generar un tejido social.

\subsection{La lucha frente al cambio climático}

El cambio climático es una realidad, su génesis se debe en gran medida a los desarrollos económicos insostenibles de los países industrializados, por tanto a partir del acuerdo de parís en 2015 (Garín, 2017) dentro de la agenda sobre los objetivos de desarrollo sostenible, se pretende alcanzar un acuerdo global para afrontar el desafío del cambio climático, por ende, uno de los mayores retos que enfrentan las políticas públicas radica en proponer modelos de toma de decisiones que prioricen el destino del planeta. (Postigo, 2013)

La principal causa del cambio climático es el consumo de combustibles fósiles, en particular petróleo y carbón, al emitir dióxido de carbono (CO2). (Mance, 2009) Aunque desde sus orígenes el planeta ha estado en continuo cambio y transformación en torno a formas de vida y especies, según el IPCC (Panel Intergubernamental de Cambio Climático) el crecimiento acelerado del calentamiento global se le atribuye en gran medida a la actividad humana y a al mal manejo del sistema económico de los países desarrollados.

El dióxido de carbono y otros gases producen calentamiento global, lo que se denomina efecto invernadero. El incremento de dichos gases, obedece en su mayoría a la explotación indiscriminada de recursos naturales, cabe resaltar que los países en desarrollo son quienes afrontan los impactos negativos asociados al extractivismo de manera directa mientras países desarrollados generan combustibles sin tener consecuencias directas, aun así, las emisiones de gases nocivos se ha convertido en una preocupación mundial, lo que ha impulsado a las grandes potencias a alcanzar acuerdos globales dentro de los objetivos del desarrollo sostenible. (Fernández, 2012)

La degradación medioambiental que se observa en la temperatura del mar, los cambios ecosistémico que amenazan el planeta están relacionados directamente con el suministro de agua y alimentos constituyen un factor de alto riesgo para la humanidad, por tanto las posturas asumidas por los movimientos sociales proponen soluciones a partir del reconocimiento de las grandes desigualdades existentes a nivel mundial en materia de distribución de la riqueza $y$ del ingreso, como una respuesta equitativa y funcional en la disminución de las emisiones de dióxido de carbono.

La tarea de los movimientos sociales parte de hacer visibles las necesidades específicas y las circunstancias especiales de los países en desarrollo, especialmente aquellas que son particularmente vulnerables a los efectos adversos que genera el cambio climático, es así que se crea el término "justicia climática" 
cuya pretensión obedece el suministrar las líneas de argumentación en relación a las responsabilidades climáticas. (Borrás, 2016)

En ese orden de ideas la gobernanza ambiental está ligada a la toma de decisiones en materia ambiental. En el ámbito de la gobernanza del agua se centra principalmente en los desafíos relacionados con el Objetivo de Desarrollo Sostenible (ODS) $6^{8}$, para superar esos desafíos desde el ámbito local se requiere el acceso a los recursos y servicios hídricos (PNUD Programa de las Naciones Unidas para el Desarrollo, 2015) lo que ha estado haciendo la Asociación de Usuarios del Distrito de Riego de Samacá ASUSA en su territorio.

\subsection{Educación Popular Ambiental}

Uno de los cimientos para alcanzar cambios sociales significativos se centra en el valor ético- político individual. Los valores ético políticos se adquieren y transforman a partir de la información que adquiere el individuo de su entorno, por ello los valores ético- políticos en gran medida se encentran condicionados por el poder hegemónico el cual limita el acceso a la información de acuerdo a su conveniencia y reproduce un modelo único de ser humano útil a los intereses del estado, a partir de lo anterior, Paulo Freire piensa la educación como un sinónimo de diálogo en el que todos los seres humanos poseen la habilidad de transmitir $y$ adquirir información lo que le permite formarse como sujeto crítico y emancipador que estimula el proceso de concienciación colectiva y transforma los valores éticos y políticos desde lo individual hasta lo colectivo. (Freire, 2007)

A partir de los años 90's inicia el auge de los movimientos ambientalistas latinoamericanos, se puede observar un vuelco en la conciencia ambiental al determinar ciertos factores que afectan la armonía de la naturaleza y los cambios de opacidad en la situación del planeta; un

\footnotetext{
$8 \quad$ garantizar la disponibilidad y la gestión sostenible
} del agua y el saneamiento para todos aparte fundamental para el inicio y consolidación permanente de estos movimientos fueron las comunidades de origen pues son ellos quienes han orientado la conservación y respeto por la naturaleza, transformando la relación entre el pensamiento y reconstrucción ética, que incide en la aplicación de hábitos, actitudes, valores y comportamientos, pro-ambientales.

Para hacer evidentes los procesos sociales que se originan en la relación que tiene la educación popular y el medio ambiente se hace necesario el diseño de prácticas de enseñanza ambiental promovidas en diversas comunidades, principalmente del medio rural, no precisamente en las aulas promulgando que el tipo de educación que se propone incluye a cualquier tipo de comunidad en general; La educación ambiental aborda como objeto de estudio las relaciones con el medio ambiente, así se delimitan aquellos elementos que lo forman y se construyen sus objetivos como una localización que da lugar a las acciones educativas, mediadas por las relaciones personales, sociales y con el ambiente biofísico. (Flores, 2013)

Cabe resaltar que la educación popular entendida como la práctica social de una cultura contada desde escenarios de experiencias propias que generan transformación de pensamiento y lectura crítica de la realidad, en relación con la educación ambiental genera además cohesión, lo que permite el fomento de seres sociales, económicos y políticos en aspectos de dinamismo colectivo con un mismo fin, que se refiere en este caso a la protección y defensa de la naturaleza, por lo tanto la participación social cumple un papel protagónico ante el surgimiento de problemas ambientales invitando a un completo sentido de pertenencia desde la comunidad.

Por otra parte las tradiciones emancipadoras que se han logrado a través de los movimientos ambientales latinoamericanos propende en gran medida a la protección del cambio 
climático y al acceso al conocimiento de las consecuencias que se han generado debido en parte a desastres socio naturales y también a la industrialización del planeta; estos aspectos hacen que la educación popular se incorpore como un componente determinante en el ámbito sociocultural y político, generando posibilidades asequibles a poblaciones de bajos recursos sobre la importancia de cuidar y como proteger el medio ambiente, lo que lleva a relacionarse con la creación de una conciencia ambiental crítica.

Si bien el cambio climático es un fenómeno global, las regiones más vulnerables al mismo se localizan entre los trópicos o cerca de ellos, por lo que sus efectos golpean con mayor severidad a los países en desarrollo, sobre todo a aquellas comunidades pobres asentadas en zonas de alto riesgo y cuyo sustento depende de la lluvia estacional para obtener resultados de sus cosechas de agricultura de subsistencia. (Gaudiano, 2007)

En un mundo globalizado las posibilidades de la población de bajos recursos para mantenerse dentro de los rangos del "buen vivir" es una tarea de subsistencia con la que se lucha día a día, se hace cada vez más devastadora frente al consumismo neoliberal, que pretende la destrucción masiva de la cosecha de la tierra siendo un pilar de sostenimiento para esta comunidades.

\section{La educación popular en el proceso de gobernanza del agua: El caso de Samacá Boyacá}

En el presente apartado abordaremos brevemente los aspectos más relevantes en relación al estudio de caso planteado, en este marco se presenta una experiencia de educación popular que enlaza los procesos gobernanza ambiental en el contexto de las conflictividades socio ambientales del Municipio de Samacá Boyacá, a partir del desarrollo de material didáctico resultado de investigación, denominado "Participación ciudadana y gobernanza ambiental ¿cómo defender

la naturaleza?", como un instrumento de difusión y apropiación social del conocimiento que ofrece a la sociedad civil en general, una guía práctica para ejercer los distintos mecanismos de participación ciudadana en material ambiental y facilitar los procesos de gobernanza ambiental de las personas y comunidades interesadas en la protección de los ecosistemas.

\subsection{El contexto del conflicto socio ambiental de Samacá:}

El municipio de Samacá se encuentra ubicado en la provincia centro del departamento de Boyacá, cuenta con una población estimada de 19,907 habitantes (DANE, 2005), por su ubicación y al contar con ecosistemas estratégicos es un municipio con importantes recursos hídricos dentro de los que se destacan el páramo rabanal y las represas de Teatinos, Gachaneca Uno y Gachaneca Dos, que proveen el agua para consumo de Samacá y algunos municipios vecinos como Tunja.

La agricultura es la única fuente de ingresos de gran parte de la población, como economía campesina tradicional busca la satisfacción de necesidades alimentarias, el uso productivo del suelo y la generación de un excedente comercializable. Los productos que se cultivan en Samacá son la Zanahoria, la Remolacha, la Cebolla, el Trigo, la Papa y el Fríjol. (Municipio de Samacà, 2016)

Para surtir las necesidades hídricas del campesinado, propias de la explotación agrícola cuenta con un Distrito de Riego y Drenaje que comprende un área total de aproximadamente 3.020 hectáreas, divididas en dos zonas: una zona plana de 2.660 hectáreas, y una zona de tierras altas más o menos quebradas, que circundan la planicie de 800 hectáreas 
dedicadas a la agricultura de subsistencia, con un total de 1.753 predios y 1.986 usuarios. El Distrito de Riego fue trasladado a la Asociación de Usuarios del Distrito de Riego de Samacá ASUSA el 15 de octubre de 1992. (Municipio de Samacà, 2006)

La economía del municipio se nutre en gran medida de la actividad minería de carbón y coquización, actividad que genera más fuentes de empleo en el municipio se estima que un $35 \%$ de la población se relaciona directamente con estas actividades (Acosta, 2016) esto a pesar de la baja estabilidad laboral para el trabajador minero y es que la industria minera en Samacá se ha establecido y llego para quedarse, según se indica:

"El sector carbonífero produce aproximadamente 22000 toneladas de carbón mensual. Contribuye con 2400 empleos directos y 1200 empleos indirectos. Las reservas de carbón de Boyacá a diciembre de 2002 se han estimado en 170.4 millones de toneladas medidas y 682.7 millones de toneladas indicadas. El Municipio de Samacá tiene reservas de carbón estimadas en 35.7 millones de toneladas medidas y 129.9 millones de toneladas indicadas." (Municipio de Samacà, 2006)

La producción del carbón de Samacá es adquirida por grandes industrias como Milpa, Inverminsa y Colcarbón, entre otras, Cuenta con cinco Contratos de Concesión de explotación del subsuelo, concedidas por el Ministerio de Minas y Energía, en un área aproximada de 4.500 Hectáreas. Actualmente Cooprocarbon agremia a 44 asociados que cuentan con 2300 trabajadores bajo tierra.

La coexistencia de estas actividades económicas en el mismo territorio no tardaría en generar tensiones ente la comunidad especialmente por el uso del suelo, por un lado las comunidades campesinas afirman que la actividad minera ha afectado los acuíferos, y el distrito de riego que administra ASUSA lo que ha tenido implicaciones directas en la siembra de cultivos y los embalses, especialmente en la vereda Salamanca donde afirman se realizó una modificación al EOT cambiando el uso del suelo para favorecer la minera lo que perjudico en forma especial a sus habitantes más de 800 usuarios del distrito de riego. Los líderes campesinos advierten que se vienen efectuando actividades mineras en ecosistemas estratégicos como el páramo Rabanal, que el seguimiento que se hace a las licencias por parte de la Agencia Nacional Minera es realmente deficiente y que el agua se estaría filtrando en los socavones proveniente de los embalses.

Por su parte la Cooperativa Boyacense productores de Carbón de Samacá (Cooprocarbon), afirma estar cumpliendo cabalmente con la normatividad ambiental y seguimiento que han hecho los entes de control y las entidades ambientales por lo cual no aceptan los señalamientos de los agricultores.

Sin embargo y tal como lo afirma (Acosta, 2016) El deterioro ambiental producto de la industria minera del carbón es inevitable.

\subsection{Las prácticas de educación popular de ASUSA y gobernanza del agua}

La gobernanza ambiental está relacionada con el desarrollo sostenible a nivel global, nacional, regional y local, su importancia radica en incluir métodos integrales que respondan a procesos transparentes de toma de decisiones en los cuales se garantice la participación ciudadana y se apoyen en marcos normativos adecuados que faciliten este proceso. (PNUD Programa de las Naciones Unidas para el Medio Ambiente, 2016)

El concepto de gobernanza se encuentra descrito en el Programa de las Naciones Unidas para el Desarrollo Reconceptualising Governance 
(1997) en el cual se define la gobernanza como: "El conjunto de mecanismos, procesos, relaciones e instituciones mediante las cuales los ciudadanos y grupos articulan sus intereses, ejercen sus derechos y obligaciones, concilian sus diferencias." (Programa de Naciones Unidas para el Desarrollo, 1977)

Es importante precisar que la ecología política enfatiza en que los contextos sociales tienen incidencia en los roles que asumen los actores sociales y sus esquemas en el uso de los recursos naturales. Critica la tendencia en la cual no existe un proceso de dialogo concertado con la ciudadanía para que esta participe activamente en la toma de decisiones, y por el contrario se le socializan decisiones ya tomadas (Montoya , Rojas , 2016) lo que va en contra de la gobernanza ambiental entendida como un gobierno horizontal.

En ese orden de ideas la gobernanza ambiental está ligada a la toma de decisiones en materia ambiental. En el ámbito de la gobernanza del agua se centra principalmente en los desafíos relacionados con el Objetivo de Desarrollo Sostenible (ODS) $6^{9}$, para superar esos desafíos desde el ámbito local se requiere el acceso a los recursos y servicios hídricos (PNUD Programa de las Naciones Unidas para el Desarrollo, 2015) lo que ha estado haciendo la Asociación de Usuarios del Distrito de Riego de Samacá ASUSA en su territorio.

El Distrito de Riego de Samacá fue trasladado a la Asociación de Usuarios del Distrito de Riego de Samacá ASUSA el 15 de octubre de 1992 tiene actualmente 1800 usuarios potenciales. Cubre 3020 hectáreas; de las cuales atiende con riego directo 2.924 hectáreas.

A partir de ese momento ASUSA ha emprendido todo un movimiento de gobernanza del agua en Samacá, inicialmente sus actividades en relación al tema fueron austeras, pues convivir

\footnotetext{
$9 \quad$ garantizar la disponibilidad y la gestión sostenible del agua y el saneamiento para todos
}

con los impactos ambientales producto de la actividad minera eran algo cotidiano, por lo tanto sus actividades se limitaron un poco a garantizar el funcionamiento del distrito de riego ejecutando proyectos de rehabilitación y mejoramiento del Canal Teatinos que conduce el agua hacia el distrito de Riego de Samacá.

Sin embargo el escalamiento de los daños ambientales producto de la actividad minera al punto de poner en riesgo la sustentabilidad del recurso hídrico ha cuestionado la posición que se venía asumiendo tomando una posición de defensa sobre los ecosistemas de paramo y bosque altoandino que surten al distrito de riego $y$ otros acueductos rurales.

Las actividades realizadas por ASUSA en aras de proteger y conservar el recurso hídrico han sido diversas, en principio se llevó a cabo un proceso de dialogo con los usuarios del distrito de riego, en él se discutieron temas como el futuro del distrito, la afectación del recurso hídrico y la industria agrícola en el municipio así como cuál sería la posición del distrito frente a la minería de carbón.

Posteriormente se llevó a cabo todo un movimiento que elevo derechos de petición, solicitudes ante entes de control, acciones populares entre otras, permeo en medios de comunicación y redes sociales vinculando a muchos actores a su problemática incentivando un proceso de dialogo que ha llegado a esferas nacionales.

Si bien es cierto se han logrado mejoras significativas como el impulso al proyecto de construcción de la PTAR, la promoción de procesos sancionatorios por minería ilegal o la solicitud a la Agencia Nacional de Minería que permitió la construcción de mesas de trabajo para analizar la problemática, el tiempo casa y la preocupación aumenta. 
Es por ello que se propuso adelantar de forma paralela un proceso de dialogo de saberes que involucre a la población Samquense, con el fin de generar conciencia ambiental en la población a través de la educación popular

Un ejemplo del papel de la educación popular en los procesos de transformación social bajo escenarios de justicia social, tiene que ver precisamente con el empoderamiento de las comunidades en torno a los procesos de gobernanza ambiental en sus territorios, como reacción a las políticas extractivas del Estado, que promueven la sustracción de recursos naturales a escala industrial de recursos naturales.

En américa latina los movimientos sociales han ofrecido diferentes respuestas en oposición al modelo extractivista, muchas de ellas con un trasfondo pedagógico concreto: la escuela popular.

Es cierto que en principio, la actividad extractiva fue bien recibida bajo la promesa de crecimiento económico para la región. Pero con el paso de los años dicha percepción fue cambiando cuando, finalizada la actividad extractiva, las comunidades conocían los devastadores efectos ambientales que tales proyectos dejaban a su paso.

La respuesta dada por los movimientos sociales y la ciudadanía a las prácticas extractivas tiene una evidente correlación con algunas transformaciones de la idea de educación en la agenda internacional, al ser considerada como elemento promotor de los objetivos del desarrollo sostenible repuestos para el año 2030, a partir de una idea de Educación transformadora y universal, como se planteó foro mundial sobre la educación del año 2015.

De hecho, desde el foro mundial de educación de 2012, ya se venía trabajando en la idea modelo educativo dirigido al empoderamiento la ciudadanía "que pueden actuar para transformar los patrones de producción, consumo y distribución del actual modelo de desarrollo, promoviendo una conciencia ambiental" (foro mundial de educación, 2012 p. 3).

Pero también se puede advertir cómo la idea inherente a la educación popular ha permeado este tipo de procesos sociales, pues en suADN se advierte la presencia de los postulados de sujeto histórico, teoría crítica, la idea de bien común y el aprendizaje en contexto, que explican en cierta medida las trasformaciones sociales en torno a la defensa de lo común, "de lo que es de todos", como es el caso de los recursos naturales. De hecho, muchos de los movimientos sociales que promovieron consultas populares a fin de prohibir el desarrollo de proyectos extractivos en sus territorios contaban, en varios casos, con el apoyo o acompañamiento de las universidades (bien de forma institucional o bien a través de algunos de sus miembros) de la región.

Bajo las anteriores premisas y en consideración al estatuto epistemológico propio de la teoría critica que orienta la educación popular de Paulo Freile, se propuso el diseño de material pedagógico dirigido a fortalecer las capacidades de gobernanza ambiental en el municipio de Samacá Boyacá.

Para la elaboración del material se llevó a cabo un proceso de diálogo de saberes, en principio dialogamos con los directivos de ASUSA quienes nos comentaron su preocupación en relación al deterioro ambiental en su territorio, y el desconocimiento que tenían algunos de sus usuarios en relación a los impactos negativos de la minería en el medio ambiente y especialmente en los recursos hídricos, posteriormente se realizaron varias visitas en las veredas, el páramo y la zona minera, a partir de ello se identificaron las principales necesidades de la comunidad lo que originó un Instrumentos de apropiación social del conocimiento o cartilla didáctica y motivo a generar presión en el municipio para 
la conformación de las mesas temáticas en las que la comunidad junto con varias instituciones del nivel local regional y nacional estudiaran los impactos de la minería en Samacá y se tomaran medidas para conservar los recursos hídricos.

\subsection{Instrumentos de apropiación social del conocimiento}

La creciente preocupación por el cuidado y protección de los ecosistemas estratégicos ha despertado el interés de la sociedad civil en participar de forma cada vez más activa en los procesos de toma de decisiones que afectan los recursos naturales, no obstante, a veces estas iniciativas se ven limitadas por la falta de orientación en torno a los tipos de mecanismos de participación ciudadana en materia ambiental y la finalidad de cada uno de ellos, marcada por una excesiva tecnocracia que limita y cercena las dinámicas de participación ciudadana. Es por esta razón, que la Fundación Universitaria Juan de Castellanos, en asocio con la Universidad Pedagógica y Tecnológica de Colombia, elaboran y presentan el documento resultado de investigación intitulado "Participación ciudadana y gobernanza ambiental ¿cómo defender la naturaleza?".

Se trata de un instrumento de difusión y apropiación social del conocimiento que ofrece a las personas, organizaciones comunitarias, ONG y sociedad civil en general, una guía práctica para ejercer los distintos mecanismos de participación ciudadana en material ambiental y facilitar los procesos de gobernanza ambiental de las personas y comunidades interesadas en la protección, defensa y cuidado de los ecosistemas estratégicos del Departamento de Boyacá como es el caso del páramo y el bosque alto andino. Este esfuerzo interinstitucional busca apoyar los procesos de gobernanza ambiental de las personas que de forma individual o colectiva han asumido el reto altruista de proteger la naturaleza de las actividades extractivas y antrópicas que ponen en alto riesgo a diversos tipos ecosistemas estratégicos.

En este sentido, un primer paso está vinculado con la divulgación de las alternativas administrativas y judiciales que pueden asegurar una participación eficiente en las decisiones ambientales. Dicha guía está estructurada de la siguiente manera: en un primer apartado se presentan nociones generales de gobernanza y medio ambiente, en segundo lugar se abordan los instrumentos internacionales y nacionales más relevantes en torno a la protección ambiental. Posteriormente se presentan las rutas de acción en los diferentes niveles territoriales, para continuar con los mecanismos de participación ciudadana en materia ambiental, para finalizar con algunos tipos de gobernanza ambiental identificados en el Departamento de Boyacá.

Se trata de material didáctico pensado por y para la comunidad, buscando construir lazos de cohesión entre la Universidad y las necesidades más sentidas de la comunidad en el Departamento de Boyacá, resultado de un dialogo de saberes a partir de la interacción de la academia con las comunidades, grupos sociales y colectivos ambientales que han asumido el reto de defender la naturaleza en sus territorios.

Este material didáctico constituye la primera experiencia de educación popular dirigida a una de las preocupaciones más relevantes en el Departamento de Boyacá, pues solo a través de este camino, el de "la revolución de las pequeñas cosas", es posible lograr la transformación con justicia social que requiere nuestro país, donde el único camino es la educación.

\section{Conclusiones}

1 El estudio de caso nos permitió analizar la aplicación de la educación popular como forma de empoderamiento de las comunidades en el tema específico de gobernanza ambiental a partir del dialogo de saberes en relación a la 
extracción minera y la conservación de las fuentes hídricas dentro del distrito de riego asociación de usuarios del distrito de adecuación de tierras de Samacá - ASUSA.

2. El estudio de caso visibilizó la trascendencia de la problemática y obligo a las autoridades a responder a las solicitudes de la comunidad, se puede decir que la experiencia de educación popular dinamizó el movimiento ambiental en defensa del agua en el municipio de Samacá.

3. Para alcanzar la construcción colectiva de relaciones exitosas entre sociedad y naturaleza, es indispensable la educación popular como alternativa para reflexionar sobre los problemas $y$ las soluciones entorno a la protección y defensa del medio ambiente.

3. Las iniciativas que surgen a partir de los movimientos ambientalistas latinoamericanos por la lucha para la conservación de los recursos naturales generan una divulgación abierta al debate manteniendo las relaciones entre la educación popular para todos y el medio ambiente.

4. La lectura crítica de la realidad a través de la educación popular ambiental genera sujetos consientes de los problemas del medio ambiente y a su vez empoderamiento por defender lo recursos naturales lo que permite la reconstrucción del tejido social.

5 La tarea con el compromiso ético y político de educar desde la experiencia, en un mundo globalizado y limitado en oportunidades para los oprimidos, hace un llamado a la transformación social desde sus bases donde la educación juega un papel trascendente.

6. Introducir el método socrático al dialogo de saberes en relación a la educación ambiental genera critica, lo que permite analizary reflexionar acerca de los procesos sociales, económicos y políticos transformando la conciencia ambiental.

\section{REFERENCIAS BIBLIOGRÁFICAS}

Acosta, D. M. (2016). Impactos ambientales de la mineria de carbón y su relación con los problemas de salud de la población del municipio de samacá (boyacá), según reportes asiS 2005-2011. Bogotà: Universidad Distrital Francisco José de Caldas.

Arciga, B. (2007). La Enseñanza Superior Como Una Práctica socio- Cultural. Revista De La Educación Superior.

Borrás, S. (2016). Movimientos para la justicia climática global: replanteando el escenario internacional del cambio climático. Grupo de Estudios de Relaciones Internacionales (GERI) , 106-110.

Calixto, F. R. (2010). Educación Popular Ambiental. Trayectorias, 31.

Cárdenas, J. F. (2018). Estudio delimitación del ecosistema de páramo y su transición hacia el bosque en los títulos mineros de la empresa Cooprocarbón - Samacá, Boyacá.

DANE. (2005). Censo General.

Fernández, J. L. (2012). El Cambio Climático: Sus Causas y Efectos Medioambientales. Anales de la Real Academia de Medicina y Cirugía de Valladolid.

Flores, R. C. (2013). Diálogos entre la pedagogía y La Educación Ambiental. Educación $Y$ Desarrollo Social, 96-99.

Freire, P. (1988). Pedagogía del oprimido. Madrid: Siglo XXI.

Freire, P. (1996). Política y Educación. Mexico: Siglo XXI Editores S.A. 
Freire, P. (2001). Política y Educación. Mexico: Siglo XXI Editores S.A.

Freire, P. (2006). Pedagogía de la autonomía: saberes necesarios para la práctica educativa. Mexico: Siglo XXI Editores S.A.

Freire, P. (2007). La educación como práctica de la libertad. Mexico: Siglo XXI Editores S.A.

Garín, A. L. (2017). Novedades del Sistema de Protección Internacional De Cambio Climático El Acuerdo De París. Instituto de Estudios Internacionales Universidad de Chile.

Gaudiano, E. G. (2007). Educación y Cambio Climático: Undesafío Inexorable. Trayectorias, 35-36.

Jiménez, M. R. (2014). Jiménez, M. R. (201 La Educación Popular En EI Siglo XXI: Una Resistencia Intercultural Desde El Sur y Desde Abajo. Praxis y Saber.

Mance, M. R. (2009). Cambio Climático: Lo Que Está En Juego. Bogotá (Colombia).

Montoya , Rojas . (2016). Elementos sobre la gobernanza y la gobernanza ambiental. Gestión y Ambiente, 302-317.

Municipio de Samacà. (2006). Plan de Desarrollo - Samaca 2004-2007 ANEXO 1.

Municipio de Samacà. (2016). http:// samacaboyaca.micolombiadigital. gov.co/si. Obtenido de http:// samacaboyaca.micolombiadigital. gov.co/sites/samacaboyaca/ content/files/000021/1028 acuerdomunicipalno009 plandedesarrollo011.pdf

Municipio de Samacà. (2016). www. samacaboyaca.micolombiadigital.gov. co. Obtenido de http://samacaboyaca. micolombiadigital.gov.co/sites/ samacaboyaca/content/files/000021/10 28acuerdomunicipalno009plandedesarr ollo011.pdf

Muñoz, D. A.n. (2017). Paulo Freire en la educación popular latinoamericana: el porqué y el para qué de estarse formando como pueblo político. Revista Kavilando.

PNUD Programa de las Naciones Unidas para el Desarrollo. (2015). La Gobernanza del Agua y los Oceanos .

PNUD Programa de las Naciones Unidas para el Medio Ambiente. (2016). www.pnuma. org. Recuperado el 26 de 02 de 2019, de http://www.pnuma.org/gobernanza/ index.php

Postigo, J. C. (2013). Cambio Climático: Movimientos sociales y Politicas Públicas. Santiago de Chile.

Programa de Naciones Unidas para el Desarrollo. (1977). Reconceptualising Governance. Nueva York .

UNDESCO, Foro Mundial de Educación. (2012). 3 